\title{
Heart Rate Variability Among Children With Acquired Brain Injury
}

\author{
Seong Woo Kim, $\mathrm{MD}^{1}$, Ha Ra Jeon, $\mathrm{MD}^{1}$, Ji Yong Kim, $\mathrm{MD}^{2}$, Yoon Kim, MD ${ }^{1}$ \\ ${ }^{1}$ Department of Physical Medicine and Rehabilitation, National Health Insurance Service Ilsan Hospital, Goyang; \\ ${ }^{2}$ Department of Physical Medicine and Rehabilitation, Inje University Ilsan Paik Hospital, Goyang, Korea
}

\begin{abstract}
Objective To find evidence of autonomic imbalance and present the heart rate variability (HRV) parameters that reflect the severity of paroxysmal sympathetic hyperactivity (PSH) in children with acquired brain injury (ABI). Methods Thirteen children with ABI were enrolled and age- and sex-matched children with cerebral palsy were selected as the control group $(n=13)$. The following HRV parameters were calculated: time-domain indices including the mean heart rate, standard deviation of all average R-R intervals (SDNN), root mean square of the successive differences (RMSSD), physical stress index (PSI), approximate entropy (ApEn); successive R-R interval difference (SRD), and frequency domain indices including total power (TP), high frequency (HF), low frequency (LF), normalized HF, normalized LF, and LF/HF ratio.

Results There were significant differences between the ABI and control groups in the mean heart rate, RMSSD, PSI and all indices of the frequency domain analysis. The mean heart rate, PSI, normalized LF, and LF/HF ratio increased in the ABI group. The presence of PSH symptoms in the ABI group demonstrated a statistically significant decline of the SDNN, TP, ln TP.

Conclusion The differences in the HRV parameters and presence of PSH symptoms are noted among ABI children compared to an age- and sex-matched control group with cerebral palsy. Within the ABI group, the presence of PSH symptoms influenced the parameters of HRV such as SDNN, TP and ln TP.
\end{abstract}

Keywords Brain injuries, Autonomic nervous system, Heart rate, Sympathetic nervous system, Parasympathetic nervous system

\section{INTRODUCTION}

The immature brain of children is extremely vulnerable to internal and external insults such as ischemia, hypoxia, traumatic force and infection. Such insults can lead to acquired brain injury (ABI) in children and cause disability or even death. Annually, over 500,000 children between birth through the age of 14 years visit emergency

Received February 20, 2017; Accepted May 17, 2017

Corresponding author: Yoon Kim

Department of Physical Medicine and Rehabilitation, National Health Insurance Service Ilsan Hospital, 100 Ilsan-ro, Ilsandong-gu, Goyang 10444, Korea. Tel: +82-31-900-0671, Fax: +82-31-900-0028, E-mail: cil36@naver.com

ORCID: Seong Woo Kim (http://orcid.org/0000-0002-1548-8147); Ha Ra Jeon (http://orcid.org/0000-0002-4234-8086); Ji Yong Kim (http://orcid. org/0000-0003-4693-8400); Yoon Kim (http://orcid.org/0000-0002-7404-8432).

(c) This is an open-access article distributed under the terms of the Creative Commons Attribution Non-Commercial License (http://creativecommons.org/ licenses/by-nc/4.0) which permits unrestricted noncommercial use, distribution, and reproduction in any medium, provided the original work is properly cited. Copyright (C) 2017 by Korean Academy of Rehabilitation Medicine 
rooms for ABI across the United States [1]. Among these cases, traumatic brain injury (TBI) is the most common cause of disability and death in children with an estimated prevalence of 475,000 cases per year in the United States $[2,3]$.

Paroxysmal sympathetic hyperactivity (PSH) after ABI is described as an increased body temperature of unknown origin, paroxysmal increase in blood pressure (BP), respiratory rate (RR), and heart rate (HR), and muscle rigidity and hyperhidrosis [4]. Since symptoms of PSH resemble nonspecific symptoms of other general medical conditions such as infection, it is often underestimated and result in unnecessary, repetitive laboratory evaluations.

Such abnormal symptoms occur in approximately $8 \%-10.5 \%$ of severe TBI patients during the acute phase [5-7]. Usually, symptoms of PSH subside within the first few weeks after injury; however, a small group of patients continue to experience episodes of PSH [8]. Recently, some studies reported PSH in pediatric patients with hypoxic ischemic encephalopathy $[9,10]$.

Clinicians tend to regard autonomic dysfunction as a minor problem; however, PSH deteriorates the clinical and functional well-being of brain injury patients. Increased morbidity that was caused by abnormal hyperthermia, catabolism and spasticity has been reported in untreated PSH patients [11,12]. Despite increasing clinical reports of this condition, it is still under-recognized; therefore, consensus on the diagnosis, evaluation and treatment of PSH in ABI children has not yet occurred.

In spite of the clinical importance of PSH, it is difficult to evaluate one's autonomic imbalance. Heart rate variability (HRV) is a non-invasive marker of cardiac autonomic function extracted from continuous electrocardiogram (ECG) recordings, which are also an emerging method to evaluate the sympathetic and parasympathetic balance of the autonomic nervous system (ANS) [13].

The aim of this study was to find evidence of autonomic imbalance and present the HRV parameters that reflect the severity of PSH in children with ABI.

\section{MATERIALS AND METHODS}

\section{Subjects}

A total of 13 children with ABI below the age of 18 years who had been admitted at the Department of Physical Medicine and Rehabilitation from October 2013 to
January 2016 were enrolled in this study. The exclusion criteria were (1) history of cardiac disease, (2) diabetes or other peripheral neurological impairment, (3) patients taking cardiovascular medications, and (4) sepsis or suspicion of significant infection $[5,14]$. To meet the objectives of this study, 13 age- and sex-matched patients with cerebral palsy were selected as the control group. Patients' demographics and etiology were obtained from their electrical medical records.

For the purposes of this study, the criteria of PSH that was defined by Baguley et al. [15] was modified. PSH was defined as any simultaneous, paroxysmal increase in at least two out of the four reported clinical data of dysautonomia (i.e., HR, RR, temperature, systolic BP [SBP]), immediately after the onset of dystonia or sweating [15]. $\mathrm{HR}, \mathrm{RR}, \mathrm{SBP}$, and axillary temperature data were collected at the occurrence of dystonia or sweating three consecutive times in 10-minute intervals and the mean value was calculated. Based on the normal HR and RR values for healthy children from birth to 18 years of age and the abnormal HR and RR criteria of advanced pediatric life support [16], abnormal HR and RR were assessed. Axillary temperature was measured using standardized digital thermometry [17]. According to the nationwide data of normal BP levels of children from 1999 to 2000 from the National Heart, Lung and Blood Institute (NHLBI) in the United States, findings above the 95th percentile are defined as abnormal [18]. Dystonia was defined as sudden occurrence or aggravation of rigidity or decerebrated posturing.

\section{HRV measurement}

HRV was analyzed using a SA-6000 device (Medicore Co., Seoul, Korea). HRV recordings were obtained for 5 minutes. Tests were conducted in a noise-free environment after 30 minutes of rest. All study participants attached electric patches to both wrists and the left ankle and remained in a relaxed supine position. In order to avoid the influence of circadian changes in the HRV results, data was collected between 1:00 PM and 3:00 PM in every patient. The room temperature during data collection was $24^{\circ} \mathrm{C}-26^{\circ} \mathrm{C}$. ECGs were obtained through 5-minute ECG recordings using personal computers. Abnormal beats, significant pauses, and areas of artifact were automatically rejected by using a computerized algorithm. After filtering, the HRV was automatically analyzed by 
the time and frequency domains. Using the fast Fourier transform (FFT), the HRV results were categorized into different frequency domains [19].

The time domain measures that were analyzed include the following: the mean heart rate, standard deviations of all average R-R intervals (SDNN), square root of the mean squared differences of successive R-R intervals (RMSSD), physical stress index (PSI), approximate entropy (ApEn), and successive R-R interval difference (SRD). The frequency domain measures included the following: total power (TP), low frequency component (LF, 0.04-0.15 Hz), high frequency component (HF, 0.15-0.4 Hz), and low to high frequency ratio (LF/HF ratio). The SDNN reflects every cyclic aspect that was related to the variability during the recording period. High SDNN values indicate irregular variability of the heart rate; while low values indicate a decreased variation of the heart rate despite an environmental change [20]. The RMSSD estimates the HF variations in heart rate, which represents the parasympathetic regulation of the heart [21]. The PSI detects the degree of load and pressure on the ANS control system. The ApEN is a measure of regularity versus randomness and quantifies the unpredictable variation of the heart rate. A low ApEN value indicates a regular signal in the test. The SRD is measured by comparing the initial to the successive part of the total measurement. The full scale value is 1 , which reflects the constant status during the measurement. Similar to the SDNN of the time domain, the TP of the frequency domain reflects the variability of the R-R intervals [20]. The LF component of the HRV is mediated by both the sympathetic and parasympathetic nerve activities, but reflects sympathetic activity more dominantly. In contrast, the HF component is mediated almost entirely by the parasympathetic nerve activity. The LF/HF ratio verifies the balance between the sympathetic and parasympathetic nerve activities. The LF and HF values were collected as normalized LF and normalized HF because of time limitations.

\section{Statistical analysis}

Data were analyzed with the chi-square test or Fisher exact test for categorical data (i.e., sex, comorbid conditions, criteria of PSH) and the Wilcoxon rank sum test for continuous data (i.e., age, study onset, HRV parameters). The data were analyzed using the Statistical Analysis Software ver. 9.2 (SAS Inc., Cary, NC, USA). All data were re- ported as a mean (minimum-maximum) for continuous variables and a number (\%) for categorical variables. For all analyses, $\mathrm{p}$-values of less than 0.05 were considered statistically significant.

\section{RESULTS}

\section{Demographics}

This study consisted of 13 children with $\mathrm{ABI}$ and 13 sexand age-matched controls with cerebral palsy. The most common etiology of the ABI group was hypoxic ischemic injury, followed by TBI and neoplasm. Among the control group, 6 were born premature and 7 were born at fullterm (Table 1). In the ABI group, the median age was 7.2 years and the median duration from brain injury to HRV measurement was 535 days. The ABI group displayed

Table 1. Etiologies and locations of brain lesions in the $\mathrm{ABI}$ and control groups

\begin{tabular}{|ccc}
\hline & $\begin{array}{c}\text { ABI group } \\
(\mathbf{n}=\mathbf{1 3})\end{array}$ & $\begin{array}{c}\text { Control group } \\
(\mathbf{n}=\mathbf{1 3})\end{array}$ \\
\hline Hypoxic ischemic injury & $7(53.85)$ & - \\
\hline Global injury & 5 & - \\
\hline Cortical injury & 1 & - \\
\hline Subcortical injury & 1 & - \\
\hline Traumatic brain injury & $4(30.77)$ & - \\
\hline Global injury & 2 & - \\
\hline Cortical injury & 1 & - \\
\hline Subcortical injury & 1 & - \\
Neoplasm & $2(15.38)$ & - \\
\hline Global injury & 2 & - \\
\hline Cortical injury & 0 & - \\
\hline Subcortical injury & 0 & - \\
\hline Preterm & - & $6(46.15)$ \\
\hline Periventricular white & - & 5 \\
matter lesion & & 0 \\
\hline Cortical lesion & - & 1 \\
\hline Subcortex involvement & - & $7(53.85)$ \\
\hline Term & - & 4 \\
\hline Periventricular white & - & 1 \\
\hline matter lesion & & 2 \\
\hline Cortical lesion & - & \\
\hline Subcortex involvement & - & \\
\hline
\end{tabular}

Values are presented as number (\%).

$\mathrm{ABI}$, acquired brain injury. 
Table 2. Demographics and comorbid conditions of the ABI and control groups

\begin{tabular}{|lccc}
\hline & ABI group (n=13) & Control group (n=13) & p-value \\
\hline Age (yr) & $7.2(1.8-16.0)$ & $7.5(1.9-16.0)$ & 0.8172 \\
\hline Sex & & & \\
\hline Male & $8(61.54)$ & $8(61.54)$ & \\
\hline Female & $5(38.46)$ & $5(38.46)$ & $0.0455^{*}$ \\
\hline Days from brain injury & $535(49-1,458)$ & $880(326-6,177)$ & $<0.0001^{*}$ \\
\hline GMFCS & $5(4-5)$ & $2(1-3)$ & $<0.0001^{*}$ \\
\hline CRS & $6(0-11)$ & $23(23-23)$ & $0.0499^{*}$ \\
\hline History of epilepsy & $9(69.23)$ & $4(30.77)$ & $0.0183^{*}$ \\
\hline Anticonvulsant & $10(76.92)$ & $4(30.77)$ & 1.0000 \\
\hline Brain pill & $2(15.38)$ & $1(7.69)$ & $0.0013^{*}$ \\
\hline Antispastic medication & $9(69.23)$ & $1(7.69)$ & 1.0000 \\
\hline Complication & & & 1.0000 \\
\hline Cardiac & $0(0)$ & $1(7.69)$ & 0.0957 \\
\hline Pulmonary & $1(7.69)$ & $0(0)$ & $0.0016^{*}$ \\
\hline Endocrine & $4(30.77)$ & $0(0)$ & 0.2200 \\
\hline Gastrointestinal & $8(61.54)$ & $0(0)$ & 0.4800 \\
\hline Modified diet & $3(23.08)$ & $0(0)$ & 0.4800 \\
\hline Genitourinary & $2(15.38)$ & $0(0)$ & 1.0000 \\
\hline Psychiatric & $2(15.38)$ & $0(0)$ & \\
\hline Musculoskeletal & $4(30.77)$ & $4(30.77)$ & \\
\hline
\end{tabular}

Values are presented as mean (minimum-maximum) or number (\%).

ABI, acquired brain injury; GMCFS, Gross Motor Function Classification System; CRS, Coma Recovery Scale. ${ }^{*} \mathrm{p}<0.05$ according to the chi-square tests, Fisher exact tests, or Wilcoxon rank sum tests.

significantly higher Gross Motor Function Classification System (GMFCS) levels and lower Coma Recovery Scale (CRS) scores than the control group ( $\mathrm{p}<0.0001$ and $\mathrm{p}=0.0001$, respectively) (Table 2 ).

\section{Comorbid condition}

The ABI and control group were significantly different in several comorbid conditions: history of epilepsy, usage of anticonvulsant, modified diet and abnormal bladder function. Among the ABI group, $69.23 \%$ had a seizure history ( $\mathrm{p}=0.0499)$, and consequently, $76.92 \%$ were on anti-epileptic drugs $(\mathrm{p}=0.0183)$. Nine patients $(69.23 \%)$ were on spasticity medication $(\mathrm{p}=0.0013)$ and 8 patients $(61.54 \%)$ were on a semi-liquid diet via L-tube or gastrostomy tube in the ABI group ( $\mathrm{p}=0.0016)$. Other comorbid conditions such as complications of the cardiac, pulmonary, endocrine, genitourinary, psychiatric, and musculoskeletal systems demonstrated no significant differences between the two groups (Table 2).

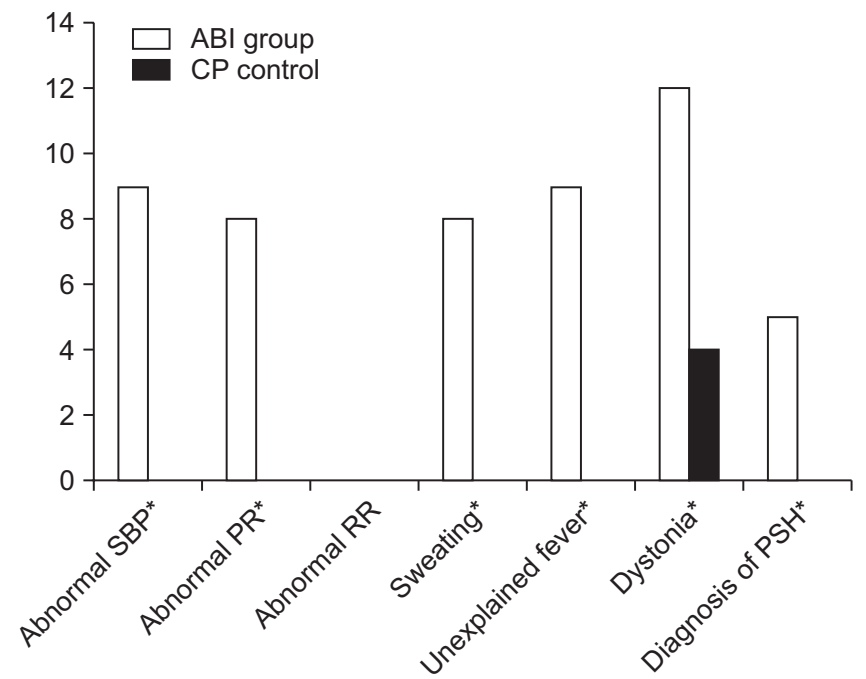

Fig. 1. Comparison of paroxysmal sympathetic hyperactivity (PSH) symptoms between the ABI and control groups. ABI, acquired brain injury; SBP, systolic blood pressure. ${ }^{*} p<0.05$ by chi-square test or Fisher exact test. 
Comparison of PSH symptoms between the $\mathrm{ABI}$ and control groups

PSH was diagnosed in 5 patients $(38.46 \%)$ in the ABI group; however, no one was diagnosed with such in the control group ( $\mathrm{p}=0.0391$ ). To be specific, five categories were significantly higher in the ABI group: abnormal SBP, abnormal PR, sweating, unexplained fever, and dystonia $(\mathrm{p}<0.0001, \mathrm{p}=0.0016, \mathrm{p}=0.0016, \mathrm{p}<0.0001$, and $\mathrm{p}=0.0013$, respectively) (Fig. 1).

Comparison of HRV between the ABI and control groups Time domain analysis

The statistical analysis demonstrated a statistically significant increase in the mean heart rate of the ABI group compared to the control group $(\mathrm{p}=0.0119)$. According to the time domain analysis, the RMSSD and PSI were significantly different between the two groups ( $\mathrm{p}=0.0240$ and $\mathrm{p}=0.0354$, respectively) (Table 3 ).

Frequency domain analysis

All indices of the frequency domain analysis demonstra- ted a statistically significant difference between the two groups. The TP, ln TP, LF, ln LF, HF, ln HF, and normalized HF significantly decreased in the ABI group compared to the control group. Only the normalized LF and LF/HF ratio increased in the ABI group compared to the control group ( $\mathrm{p}=0.0018$ and $\mathrm{p}=0.0018$, respectively) (Table 3 ).

\section{Comparison of HRV between the PSH and non-PSH patients among the $A B I$ group \\ Time domain analysis}

Among the ABI groups, 5 patients were diagnosed with PSH (PSH-ABI group), while 8 patients did not meet the criteria of PSH (non-PSH-ABI group). The SDNN values in the PSH-ABI group significantly decreased compared to the non-PSH-ABI group ( $\mathrm{p}=0.0134)$. The remainder of the time domain analysis resulted in no other significant differences between the two subgroups (Table 4).

\section{Frequency domain analysis}

The TP and ln TP significantly decreased in the PSH-ABI group compared to the non-PSH-ABI group ( $\mathrm{p}=0.0043$,

Table 3. Comparison of HRV between the ABI and control groups

\begin{tabular}{|lccl|}
\hline & ABI group $(\mathbf{n}=\mathbf{1 3})$ & Control group $(\mathbf{n}=\mathbf{1 3})$ & p-value \\
\hline Time domain & & & \\
\hline Heart rate & $111(95-149)$ & $97(58-133)$ & $0.0119^{*}$ \\
\hline SDNN (ms) & $25.916(9.276-73.36)$ & $47.447(21.309-57.680)$ & 0.1509 \\
\hline RMSSD (ms) & $17.208(9.290-48.531)$ & $42.361(12.231-61.437)$ & $0.0240^{*}$ \\
\hline PSI & $129.673(23.908-606.616)$ & $37.403(16.132-204.967)$ & $0.0354^{*}$ \\
\hline ApEn & $1.032(0.040-1.309)$ & $1.104(0.724-1.249)$ & 0.9591 \\
\hline SRD & $1.077(0.744-1.418)$ & $1.000(0.604-1.222)$ & 0.1996 \\
\hline Frequency domain & & & $0.0312^{*}$ \\
\hline TP $\left(\mathrm{ms}^{2}\right)$ & $406.574(56.543-5,423.10)$ & $1581.000(314.867-3,167.61)$ & $0.0312^{*}$ \\
\hline ln TP & $6.008(4.035-8.598)$ & $7.366(5.752-8.061)$ & $0.0210^{*}$ \\
\hline LF $\left(\mathrm{ms}^{2}\right)$ & $72.431(16.917-952.924)$ & $296.300(93.167-964.410)$ & $0.0210^{*}$ \\
\hline ln LF & $4.238(2.828-6.960)$ & $5.691(4.535-6.872)$ & $0.0009^{*}$ \\
\hline HF $\left(\mathrm{ms}^{2}\right)$ & $51.063(1.836-237.086)$ & $271.360(94.389-1,062.63)$ & $0.0009^{*}$ \\
\hline ln HF & $3.933(0.607-5.468)$ & $5.603(4.547-6.969)$ & $0.0018^{*}$ \\
\hline Normalized LF & $69.558(25.659-90.212)$ & $48.807(29.913-64.794)$ & $0.0018^{*}$ \\
\hline Normalized HF & $30.442(9.788-74.341)$ & $51.193(35.206-70.087)$ & $0.0018^{*}$ \\
\hline LF/HF ratio & $2.285(0.345-9.217)$ & $0.953(0.427-1.840)$ & \\
\hline
\end{tabular}

Values are presented as mean (minimum-maximum).

HRV, heart rate variability; ABI, acquired brain injury; SDNN, standard deviations of normal to normal R-R intervals; RMSSD, square root of the sum of the square of differences between adjacent R-R intervals; PSI, physical stress index; ApEn, approximate entropy; SRD, successive R-R interval difference; TP, total power; LF, low frequency; HF, high frequency.

${ }^{*} \mathrm{p}<0.05$ according to the Wilcoxon rank sum test. 
Table 4. Comparison of HRV between PSH and non-PSH patients in the ABI group

\begin{tabular}{|lccc}
\hline & PSH patients (n=5) & Non-PSH patients (n=8) & p-value \\
\hline Time domain & & & \\
\hline Heart rate & $119(105-149)$ & $108(95-138)$ & 0.2121 \\
\hline SDNN (ms) & $22.592(9.276-27.098)$ & $45.785(18.967-73.360)$ & $0.0134^{*}$ \\
\hline RMSSD (ms) & $14.672(9.290-24.479)$ & $25.138(10.019-48.531)$ & 0.1643 \\
\hline PSI & $137.153(115.100-606.616)$ & $59.884(23.908-217.046)$ & 0.2723 \\
\hline ApEn & $1.161(0.945-1.309)$ & $1.024(0.040-1.203)$ & 0.4208 \\
\hline SRD & $1.154(1.015-1.240)$ & $1.013(0.744-1.418)$ & 0.5101 \\
\hline Frequency domain & & & $0.0043^{*}$ \\
\hline TP $\left(\mathrm{ms}^{2}\right)$ & $250.307(56.543-326.085)$ & $1095.69(399.508-5,423.10)$ & $0.0043^{*}$ \\
\hline ln TP & $5.523(4.035-5.787)$ & $6.990(5.990-8.598)$ & 0.0923 \\
\hline LF $\left(\mathrm{ms}^{2}\right)$ & $52.132(33.165-130.748)$ & $253.896(16.917-952.924)$ & 0.0923 \\
\hline ln LF & $3.954(3.501-4.873)$ & $5.531(2.828-6.960)$ & 0.4208 \\
\hline HF $\left(\mathrm{ms}^{2}\right)$ & $22.815(6.315-191.584)$ & $131.161(1.836-237.086)$ & 0.4208 \\
\hline ln HF & $3.127(1.843-5.255)$ & $4.876(0.607-5.468)$ & 1.000 \\
\hline Normalized LF & $71.914(25.659-84.004)$ & $68.483(48.807-90.212)$ & 1.000 \\
\hline Normalized HF & $28.086(15.996-74.341)$ & $31.5175(9.788-51.193)$ & 1.000 \\
\hline LF/HF ratio & $2.561(0.345-5.251)$ & $2.173(0.953-9.217)$ & \\
\hline
\end{tabular}

Values are presented as mean (minimum-maximum).

HRV, heart rate variability; PSH, paroxysmal sympathetic hyperactivity; ABI, acquired brain injury; SDNN, standard deviations of normal to normal R-R intervals; RMSSD, square root of the sum of the square of differences between adjacent R-R intervals; PSI, physical stress index; ApEn, approximate entropy; SRD, successive R-R interval difference; TP, total power; LF, low frequency; HF, high frequency.

${ }^{*} \mathrm{p}<0.05$ according to the Wilcoxon rank sum test.

$\mathrm{p}=0.0043, \mathrm{p}=0.0043$, and $\mathrm{p}=0.0043$, respectively). Other frequency domain parameters displayed no other significant differences between the two subgroups (Table 4).

Comparison of PSH symptoms between the subgroups based on the location of the brain lesion in the $A B I$ and control groups

The ABI group can be divided into three subgroups according to the location and range of the brain lesion: global $(n=9)$, cortical $(n=2)$, and subcortical injury $(n=2)$ [22]. Similarly, the control group can be divided into three subgroups according to the location of the brain lesion: periventricular $(n=9)$, cortical $(n=1)$, and subcortical lesion ( $n=3)$. The occurrence of each PSH symptom and diagnosis of PSH were not significantly different between each of the subgroups based on the location of the brain lesion. A trend of a higher diagnostic rate of PSH was noted among global injury subgroups in the ABI group (Table 5).
Table 5. PSH according to the location of the brain lesion in the ABI and control groups

\begin{tabular}{lll}
\hline & PSH & Non-PSH \\
\hline ABI group (n=13) & & \\
Global injury & $4(30.77)$ & $5(38.46)$ \\
Cortical injury & $1(7.69)$ & $1(7.69)$ \\
Subcortical injury & $0(0.00)$ & $2(15.38)$ \\
Control group (n=13) & & \\
Periventricular white matter lesion & $0(0.00)$ & $9(69.23)$ \\
Cortical lesion & $0(0.00)$ & $1(7.69)$ \\
Subcortex involvement & $0(0.00)$ & $3(23.08)$ \\
\hline
\end{tabular}

Values are presented as number (\%).

PSH, paroxysmal sympathetic hyperactivity; ABI, acquired brain injury.

\section{DISCUSSION}

Childhood ABI results in various forms of disturbances involving motor, sensory, cognitive and behavioral dysfunctions. PSH is also observed after ABI; however, it has 
rarely been studied, especially in the pediatric population. In spite of the scarcity of clinical reports, recognition of PSH is crucial because it is associated with increased morbidity rates and poorer outcomes $[6,8,23,24]$.

Many scholars have presented various hypotheses regarding the pathophysiology of PSH after brain injury. Two mainstream hypotheses have been settled: a theory inferring an epileptogenic cause and a theory involving the disconnection syndrome, which suggests injury to an extensive brain lesion from the cerebral cortex, diencephalon to the upper brainstem and medulla [5]. Based on the epileptogenic theory, previous studies have attempted to detect and treat epilepsy in patients suffering from PSH, though the results were negative [25-27]. Disconnection syndromes are thought to occur by either mechanical damage or a neurotransmitter blockade. One study supported the disconnection syndrome theory by reporting markedly variable LF/HF ratios and decreased LF power in severe TBI patients with PSH compared to patients without PSH [5]. In spite of such efforts, direct evidence of the disconnection syndrome is still lacking $[4,28]$. The excitatory/inhibitory ratio (EIR) model was recently presented as a modified version of the disconnection theory to explain diverse severe autonomic overactivity after brain injury, such as neuroleptic malignant syndrome, serotonin syndrome, parkinsonian-hyperpyrexia syndrome, malignant hyperthermia, and PSH. In this model, the autonomic overactivity is explained by the damaged diencephalic/brainstem inhibitory center which abnormally converts the non-nociceptive afferent stimuli from the spinal cord to nociceptive input and consequently increases the sympathetic excitatory activity [29]. The aim of this study was to prove the abnormal EIR of the ANS and identify the HRV parameters that reflect the autonomic dysfunction of ABI children with PSH compared to age- and sex-matched control children with cerebral palsy.

In this study, the ABI group, which had significantly higher GMFCS levels and lower CRS scores, displayed a higher rate of abnormal SBP, PR, sweating, unexplained fever and dystonia, which resulted in a significantly higher diagnostic rate of PSH. Compared to the control group, the $\mathrm{ABI}$ group revealed significant increases in the mean heart rate and PSI, and a significant decrease in TP, which emphasizes the reduction of the ANS in a dynamic complexity. Moreover, the significant decline of the RMS-
SD, LF, HF, normalized HF and significant incline of the normalized LF, LF/HF ratio in the ABI group reflected the imbalance between the sympathetic and parasympathetic systems. Since the LF reflected the sympathetic activity more dominantly, the significantly increased normalized LF of the ABI group indicated high sympathetic excitatory activity. While HF reflected the parasympathetic activity, the significantly decreased HF and normalized HF values of the ABI group implied low parasympathetic activity. The significant increase of the LF/HF ratio of the ABI group mirrored the stronger influence of the sympathetic power over parasympathetic power. As a result, this study demonstrated that the ABI group has higher sympathetic excitatory activity and more dominant sympathetic power than parasympathetic power compared to the control group.

Consistent with our results, previous studies involving adult traumatic brain injury patients demonstrated significantly lower LF, TP, and SDNN, and a significantly elevated LF/HF ratio $[5,30]$. One pediatric HRV study of boys who suffered severe traumatic brain injury exhibited a significantly higher heart rate at rest and during exercise compared to typically-developed controls. This study also reported a lower RMSSD of the HRV at rest and significantly decreased time-domain values during exercise only in traumatic brain injury children [31].

For clinical convenience, the measurement of vital signs (i.e., HR, RR, SBP, and temperature) that were used as the diagnostic criteria of PSH promptly after the onset of dystonia or sweating instead of continuous monitoring was used. Among the ABI patients, 5 patients (38.46\%) were diagnosed with PSH. The PSH-ABI group had significantly lower values of the SDNN and TP compared to the non-PSH-ABI group. Since the SDNN and TP values represent the irregular variability of heart rate, decreased SDNN and TP indicate a less variable nature of the heart rate during environmental change. In short, the ABI group has less adaptability of their ANS to a changing environment. Although the mean heart rate and LF/HF ratio were higher values in the PSH-ABI group, it was not statistically significant. The only previous study of HRV measurement that compared PSH and non-PSH groups in ABI suggested significantly lower LF values in adult TBI patients with PSH [5]. This difference may be due to the small sample size of the previous and current studies ( $n=16, n=13$, respectively). Nonetheless, the overall trend 
of the HRV parameters reflected a lack of adaptability of the ANS to a continuously changing environment and a predominance of sympathetic excitatory power over parasympathetic inhibitory power, which supports the EIR model [32].

There are a number of limitations of this study. First, the number of subjects included in this study was relatively small due to the relative scarcity of inpatient, ABI pediatric patients. To overcome this limitation, we conducted a sex- and age-matched case-control study. Second, the sex- and age-specific reference values of the HRV parameters have not been established, especially in children. Since the normal value of the heart rate differs among sex and age in children [33], the normal values of the HRV parameters differ. To produce a more accurate correlation of the HRV parameters and PSH, sex- and age-specific normal values of the HRV parameters are necessary. Third, the results only represented the state of the CNS heart rate control while subjects are at rest. As seen in other dynamic studies conducted at rest and during exercise [28], the HRV parameters change with provocation of the sympathetic tone compared to the resting state, and in patients with PSH, this gap is expected to maximize. Finally, each subject underwent measurement of their HRV at a different period from the onset of disease. As proved by previous studies, patients were more prone to present dysautonomia features during the earlier period after brain injury and exhibited decreased values of the HRV parameters. This different timing of the HRV measurement may influence the overall results of this study. Nevertheless, this study demonstrated that ABI children with PSH presented dominant values of the sympathetic HRV parameters at rest. Further multicenter studies with large sample sizes of HRV measurement at rest and during dysautonomia symptoms in ABI children at the same time period of post-injury is necessary.

In conclusion, the difference in the HRV parameters and presence of PSH symptoms were noted among ABI children compared to an age- and sex-matched control group with cerebral palsy. The presence of PSH symptoms in pediatric $\mathrm{ABI}$ patients influenced the parameters of HRV, especially the SDNN and TP values that represent the irregular variability of heart rate.

\section{CONFLICT OF INTEREST}

No potential conflict of interest relevant to this article was reported.

\section{REFERENCES}

1. Centers for Disease Control and Prevention. Traumatic brain injury in the United States: emergency department visits, hospitalizations, and deaths 20022006 (blue book) [Internet]. Atlanta: Centers for Disease Control and Prevention; c2016 [cited 2017 Nov 1]. Available from: http://www.cdc.gov/traumaticbraininjury/tbi_ed.html.

2. Schaller AL, Lakhani SA, Hsu BS. Pediatric traumatic brain injury. S D Med 2015;68:457-61.

3. DeMatteo CA, Hanna SE, Yousefi-Nooraie R, Lin CY, Mahoney WJ, Law MC, et al. Quality-of-life after brain injury in childhood: time, not severity, is the significant factor. Brain Inj 2014;28:114-21.

4. Baguley IJ, Cameron ID, Green AM, Slewa-Younan S, Marosszeky JE, Gurka JA. Pharmacological management of dysautonomia following traumatic brain injury. Brain Inj 2004;18:409-17.

5. Baguley IJ, Heriseanu RE, Felmingham KL, Cameron ID. Dysautonomia and heart rate variability following severe traumatic brain injury. Brain Inj 2006;20:43744.

6. Baguley IJ, Slewa-Younan S, Heriseanu RE, Nott MT, Mudaliar Y, Nayyar V. The incidence of dysautonomia and its relationship with autonomic arousal following traumatic brain injury. Brain Inj 2007;21:1175-81.

7. Hendricks HT, Geurts AC, van Ginneken BC, Heeren AJ, Vos PE. Brain injury severity and autonomic dysregulation accurately predict heterotopic ossification in patients with traumatic brain injury. Clin Rehabil 2007;21:545-53.

8. Baguley IJ, Nicholls JL, Felmingham KL, Crooks J, Gurka JA, Wade LD. Dysautonomia after traumatic brain injury: a forgotten syndrome? J Neurol Neurosurg Psychiatry 1999;67:39-43.

9. Raithel DS, Ohler KH, Porto I, Bicknese AR, Kraus DM. Morphine: an effective abortive therapy for pediatric paroxysmal sympathetic hyperactivity after hypoxic brain injury. J Pediatr Pharmacol Ther 2015;20:33540. 
10. Israeli-Mendlovic H, Mendlovic J, Katz-Leurer M. Heart rate and heart rate variability parameters at rest, during activity and passive standing among children with cerebral palsy GMFCS IV-V. Dev Neurorehabil 2014;17:398-402.

11. Scott JS, Ockey RR, Holmes GE, Varghese G. Autonomic dysfunction associated with locked-in syndrome in a child. Am J Phys Med Rehabil 1997;76:2003.

12. Lemke DM. Riding out the storm: sympathetic storming after traumatic brain injury. J Neurosci Nurs 2004; 36:4-9.

13. Jarrin DC, McGrath JJ, Poirier P, Seguin L, Tremblay RE, Montplaisir JY, et al. Short-term heart rate variability in a population-based sample of 10-year-old children. Pediatr Cardiol 2015;36:41-8.

14. Biswas AK, Scott WA, Sommerauer JF, Luckett PM. Heart rate variability after acute traumatic brain injury in children. Crit Care Med 2000;28:3907-12.

15. Baguley IJ, Perkes IE, Fernandez-Ortega JF, Rabinstein AA, Dolce G, Hendricks HT, et al. Paroxysmal sympathetic hyperactivity after acquired brain injury: consensus on conceptual definition, nomenclature, and diagnostic criteria. J Neurotrauma 2014;31:1515-20.

16. Fleming S, Thompson M, Stevens R, Heneghan C, Pluddemann A, Maconochie I, et al. Normal ranges of heart rate and respiratory rate in children from birth to 18 years of age: a systematic review of observational studies. Lancet 2011;377:1011-1018.

17. Leduc D, Woods S. Temperature measurement in paediatrics [Internet]. Ottawa: Canadian Paediatric Society; c2016 [cited 2017 Nov 1]. Available from: http:// www.cps.ca/en/documents/position/temperaturemeasurement.

18. National Heart, Lung, and Blood Institute. Blood pressure tables for children and adolescents: from the fourth report on the diagnosis, evaluation, and treatment of high blood pressure in children and adolescents. Bethesda: National Heart, Lung, and Blood Institute; 2004 [cited 2017 Nov 1]. Available from: http:// www.nhlbi.nih.gov/health-pro/guidelines/current/ hypertension-pediatric-jnc-4/blood-pressure-tables.

19. Shim SH, Park SY, Moon SN, Oh JH, Lee JY, Kim HH, et al. Baseline heart rate variability in children and adolescents with vasovagal syncope. Korean J Pediatr 2014;57:193-8.
20. Task Force of the European Society of Cardiology and the North American Society of Pacing and Electrophysiology. Heart rate variability: standards of measurement, physiological interpretation, and clinical use. Eur Heart J 1996;17:354-81.

21. Ponnusamy A, Marques JL, Reuber M. Comparison of heart rate variability parameters during complex partial seizures and psychogenic nonepileptic seizures. Epilepsia 2012;53:1314-21.

22. Benarroch EE. The central autonomic network: functional organization, dysfunction, and perspective. Mayo Clin Proc 1993;68:988-1001.

23. Lv LQ, Hou LJ, Yu MK, Qi XQ, Chen HR, Chen JX, et al. Prognostic influence and magnetic resonance imaging findings in paroxysmal sympathetic hyperactivity after severe traumatic brain injury. J Neurotrauma 2010;27:1945-50.

24. Pignolo L, Rogano S, Quintieri M, Leto E, Dolce G. Decreasing incidence of paroxysmal sympathetic hyperactivity syndrome in the vegetative state. J Rehabil Med 2012;44:502-4.

25. Baguley IJ, Heriseanu RE, Gurka JA, Nordenbo A, Cameron ID. Gabapentin in the management of dysautonomia following severe traumatic brain injury: a case series. J Neurol Neurosurg Psychiatry 2007;78: 539-41.

26. Do D, Sheen VL, Bromfield E. Treatment of paroxysmal sympathetic storm with labetalol. J Neurol Neurosurg Psychiatry 2000;69:832-3.

27. Thorley RR, Wertsch JJ, Klingbeil GE. Acute hypothalamic instability in traumatic brain injury: a case report. Arch Phys Med Rehabil 2001;82:246-9.

28. Boeve BF, Wijdicks EF, Benarroch EE, Schmidt KD. Paroxysmal sympathetic storms ("diencephalic seizures") after severe diffuse axonal head injury. Mayo Clin Proc 1998;73:148-52.

29. Baguley IJ. The excitatory:inhibitory ratio model (EIR model): an integrative explanation of acute autonomic overactivity syndromes. Med Hypotheses 2008;70:2635.

30. King ML, Lichtman SW, Seliger G, Ehert FA, Steinberg JS. Heart-rate variability in chronic traumatic brain injury. Brain Inj 1997;11:445-53.

31. Katz-Leurer M, Rotem H, Keren O, Meyer S. Heart rate and heart rate variability at rest and during exercise in boys who suffered a severe traumatic brain injury and 
typically-developed controls. Brain Inj 2010;24:110-4. 32. Baguley IJ, Heriseanu RE, Cameron ID, Nott MT, Slewa-Younan S. A critical review of the pathophysiology of dysautonomia following traumatic brain injury. Neurocrit Care 2008;8:293-300.
33. Bonafide CP, Brady PW, Keren R, Conway PH, Marsolo K, Daymont C. Development of heart and respiratory rate percentile curves for hospitalized children. Pediatrics 2013;131:e1150-7. 